\title{
Maternal nutrition induces gene expression changes in fetal muscle and adipose tissues in sheep
}

\author{
Francisco Peñagaricano ${ }^{1 *}$, Xin Wang ${ }^{2}$, Guilherme JM Rosa', Amy E Radunz ${ }^{3}$ and Hasan Khatib ${ }^{1 *}$
}

\begin{abstract}
Background: Maternal nutrition during different stages of pregnancy can induce significant changes in the structure, physiology, and metabolism of the offspring. These changes could have important implications on food animal production especially if these perturbations impact muscle and adipose tissue development. Here, we evaluated the impact of different maternal isoenergetic diets, alfalfa haylage (HY; fiber), corn (CN; starch), and dried corn distillers grains (DG; fiber plus protein plus fat), on the transcriptome of fetal muscle and adipose tissues in sheep.

Results: Prepartum diets were associated with notable gene expression changes in fetal tissues. In longissimus dorsi muscle, a total of 224 and 823 genes showed differential expression (FDR $\leq 0.05$ ) in fetuses derived from DG vs. CN and HY vs. CN maternal diets, respectively. Several of these significant genes affected myogenesis and muscle differentiation. In subcutaneous and perirenal adipose tissues, 745 and 208 genes were differentially expressed (FDR $\leq 0.05$ ), respectively, between CN and DG diets. Many of these genes are involved in adipogenesis, lipogenesis, and adipose tissue development. Pathway analysis revealed that several GO terms and KEGG pathways were enriched (FDR $\leq 0.05)$ with differentially expressed genes associated with tissue and organ development, chromatin biology, and different metabolic processes.
\end{abstract}

Conclusions: These findings provide evidence that maternal nutrition during pregnancy can alter the programming of fetal muscle and fat tissues in sheep. The ramifications of the observed gene expression changes, in terms of postnatal growth, body composition, and meat quality of the offspring, warrant future investigation.

Keywords: Maternal nutrition, Transcriptome analysis, RNA-sequencing, Fetal programming, Sheep

\section{Background}

Fetal developmental programming describes the process whereby a maternal stimulus or insult during a critical period of development has lasting or lifelong consequences [1]. Maternal nutrition is considered a major intrauterine environmental factor in fetal development. In fact, it is well established that maternal nutrition during different stages of pregnancy can induce permanent changes in the structure, physiology, and metabolism of the offspring [2,3]. Epidemiological studies in humans have shown that nutrient restriction during gestation increases the incidence of postnatal disorders, including obesity and coronary heart disease [4,5]. In addition,

\footnotetext{
* Correspondence: fpenagarican@wisc.edu; hkhatib@wisc.edu

${ }^{1}$ Department of Animal Sciences, University of Wisconsin-Madison, 1675

Observatory Drive, Madison, WI 53706, USA

Full list of author information is available at the end of the article
}

experimental studies using rodent models have shown that both maternal undernutrition and overnutrition have significant effects on fetal development with long term implications. For instance, maternal dietary restriction leads to delayed postnatal growth and elevated blood pressure of offspring [6]. Restriction of maternal protein intake throughout gestation resulted in decreased birth weight, perturbations to renin-angiotensin homeostasis, kidney disorders, and adult hypertension [7]. Maternal high-fat over-feeding during pregnancy resulted in several disorders in the offspring, including abnormal glucose homeostasis, abnormal serum lipid profiles, and increased adiposity [8]. Overall, these studies are consistent with the idea that maternal nutrition during pregnancy can induce remarkable effects on fetal development, which in turn may predispose 
the offspring to metabolic, endocrine, and cardiovascular disorders in postnatal life.

Postnatal effects of maternal nutrition on growth, productivity, and reproduction of the offspring could have important implications in the livestock industry. Indeed, evidence suggests that maternal nutrient intake can alter subsequent growth, skeletal muscle development, body composition, and energy metabolism in early postnatal life of offspring in livestock species $[9,10]$. Different prepartum maternal energy sources fed during late gestation in beef cattle resulted in changes in birth weight and postnatal muscle and fat deposition in progeny [11-14]. Similarly, prepartum diets during mid to late gestation in sheep had significant effects on postnatal carcass composition in terms of both fat and muscle deposition [15]. Dietary components, such as protein content or fat supplementation, have been associated with alterations in the offspring performance. Maternal protein supplementation during late gestation has been positively associated with postnatal growth and adipose deposition in steers (male progeny), and also postweaning body weight and fertility in heifers (female progeny) in beef cattle [16,17]. Fat supplementation in gestating ewes increased cold tolerance and improved survival of their lambs [18]. Maternal energy sources, such as starch or fiber, may also impact fetal development and subsequent performance of the offspring. Maternal starchbased diets in cattle have been associated with greater calf birth weights compared with fiber-based prepartum diets $[19,20]$. Therefore, these studies in livestock species clearly show that prepartum diets can affect the performance of the offspring, including postnatal growth, body composition, and carcass weight and may have significant implications in food animal production.

Fetal programming of skeletal muscle and adipose tissue development may have substantial effects on economic viability of livestock enterprises through impacts on efficiency of production and product quality. Skeletal muscle mass is largely determined by the number and the size of muscle fibers. Muscle fibers are formed exclusively during the prenatal stage, especially from early to mid-gestation, and there is no further net increase in the number of these fibers after birth [21]. In contrast, the size of muscle fibers depends on satellite cells which proliferate, synthesize myofibrillar proteins, and fuse with existing muscle fibers to increase muscle fiber size which starts to occur in late gestation and continues in postnatal development [21]. Therefore, programming of muscle tissue during fetal development which could lead to a lesser number and smaller size of muscle fibers would reduce muscle mass and have negative effects on efficiency of animal production [21,22]. Studies in sheep have shown that maternal undernutrition in early gestation mainly affects fetal muscle fiber number while inadequate nutrition during late gestation impacts fetal muscle fiber size [23-25]. The majority of fetal adipose tissue is developed during the final third of gestation, and it is now well established that maternal nutrition plays a key role in its development [26]. In fact, both maternal undernutrition and overnutrition can result in the long-term programming of adipose tissue abundance and function [27,28]. Ovine studies have shown that maternal nutrient restriction during early to mid-gestation, coincident with the period of maximal placental growth, results in increased perirenal adipose tissues at birth [29]. Conversely, maternal undernutrition during late gestation, which is coincident with the period of maximal fetal growth, results in reduced adiposity in the offspring [29]. Furthermore, overnutrition during late pregnancy induces overexpression of genes regulating adipogenesis and lipogenesis in fetal perirenal fat tissue in sheep, which in turn may result in increased adiposity in later life [30]. These studies show that maternal nutrition can alter the composition and abundance of fetal skeletal muscle and fat depots that may affect livestock production.

Previous studies have reported the effects of maternal nutrition on fetal development in livestock species, including fetal programming of muscle and fat tissues, using extreme undernutrition or overnutrition conditions. Few studies have evaluated the possible impact of subtle differences in maternal diets, such as different energy sources or subtle differences in protein content, on the overall expression of the fetal genome. Maternal diets containing different energy sources, such as starch, fiber or fat, or showing subtle differences in protein content, may alter fetal development in livestock species to have long term implications in food animal production. We have previously reported that maternal diets differing in fat and protein content alter postnatal growth traits in cattle and sheep $[11,15,31]$. The mechanisms, however, by which these diets affect muscle and fat tissues are not known. Additionally, our recent study has shown that the expression of imprinted genes in fetal tissues was influenced by maternal diets that differed in energy sources [32], but genome-wide expression differences have not yet been explored. As such, the objective of this study was to investigate the impact of different maternal dietary feed sources during mid-to-late gestation on the transcriptome of fetal muscle and adipose tissues in sheep.

\section{Methods}

\section{Ethics statement}

The College of Agriculture and Life Sciences Animal Care and Use Committee of the University of WisconsinMadison approved the procedures used in this study.

\section{Animals, experimental design, and maternal diets}

Multiparous Polypay ewes were used in a completely randomized design to evaluate the effect of different 
maternal diets during mid-to-late gestation on the transcriptome of different fetal tissues. Ewes were naturally bred to a single sire and from days $67 \pm 3$ of gestation until necropsy (days $130 \pm 1$ ), they were individually fed one of three diets where the primary energy source was alfalfa haylage ( $\mathrm{HY}$; fiber), corn ( $\mathrm{CN}$; starch), or dried corn distillers grains (DG; fiber, protein and fat). These diets were formulated to meet nutritional requirements of the ewes during these stages of production. Table 1 shows the daily nutrient intake of the ewes by dietary treatment during the experiment. Intake of $\mathrm{CN}$ and DG diets was limited to achieve isoenergetic intake among dietary treatments relative to an ad libitum intake of HY, and intake of $\mathrm{CN}$ and DG diets was adjusted to achieve similar body weight gains of ewes from days 67 to days 130 of gestation.

\section{Tissue collection}

Ewes were necropsied on day $130 \pm 1$ of gestation. A total of 26 fetuses were removed from 15 dams. From the fetuses three different tissues were collected including one muscle, longissimus dorsi muscle from the left side of the fetus, and two adipose tissues, perirenal adipose depot and subcutaneous adipose depot near the shoulder blade. These tissues were immediately frozen at $-80^{\circ} \mathrm{C}$ until RNA extraction was performed.

\section{RNA extraction and preparation of pooled samples}

Total RNA was extracted from tissue samples using the RNeasy Mini kit (QIAGEN, Valencia, CA, USA) and then treated with DNase-free DNase Set (QIAGEN) to avoid genomic DNA amplification. Concentrations and $\mathrm{OD}_{260 / 280}$ ratios of RNA samples were measured with the Nanodrop ND-1000 spectrophotometer (Nanodrop Technologies, Montchanin, DE).

Table 1 Daily nutrient intake of ewes from days 67 to 130 of gestation on a dry matter basis

\begin{tabular}{lccc}
\hline & \multicolumn{3}{c}{ Diets } \\
\cline { 2 - 4 } Item & HY & CN & DG \\
\hline DMl, kg/d & 2.03 & 1.17 & 1.18 \\
Alfalfa haylage & 2.03 & 0.14 & 0.17 \\
Corn & - & 0.80 & - \\
DDGS & - & - & 0.77 \\
Supplement & - & 0.23 & 0.24 \\
Nutrient intake & & & \\
Crude protein (g/d) & 383.26 & 130.63 & 309.84 \\
NDF (g/d) & 940.10 & 198.82 & 508.16 \\
Crude fat (g/d) & 85.97 & 84.94 & 114.02 \\
\hline
\end{tabular}

Diets fed to dams: $\mathbf{H Y}=$ ad libitum fed alfalfa haylage; $\mathbf{C N}=$ limit-fed whole shell corn;

DG = limit-fed corn dried distiller's grains (adapted from Lan et al. [32]).
The RNA samples from the 26 fetuses were pooled to generate four biological replicates per maternal diet and tissue. In particular, per each diet and tissue, two RNA pools were created from male fetuses and two RNA pools were created from female fetuses. The description of the pools including number of fetuses and dams can be found in Additional file 1.

\section{Library generation and RNA sequencing}

A total of $50 \mathrm{ng}$ of RNA from each pool was used to prepare sequencing libraries following Illumina's mRNASeq protocol. Libraries were sequenced with Illumina's HiSeq 2000 at the Biotechnology Center in the University of Wisconsin-Madison. The 36 libraries (i.e., 12 libraries per tissue, three tissues in total) were barcoded, multiplexed, and then sequenced. A read was defined as a $100 \mathrm{bp}$ cDNA fragment sequenced from a single end. Approximately 30 million reads were sequenced from each library.

\section{Mapping reads to the reference genome}

Raw sequence reads were mapped directly to the reference sheep genome (Oar_v3.1) using the software package Tophat (v2.0.4) [33]. A two-step approach was used in order to maximize sensitivity to splice junction discovery: the first alignment was performed in each of the samples independently, and then novel splice junctions plus known splice junctions from Ensembl annotation were combined and supplied to Tophat for a second alignment. This approach allows for full utilization of the novel junctions identified in the samples. A maximum of two mismatches were allowed and reads that mapped equally well to more than 40 genomic locations were discarded.

\section{Assembly of transcripts and estimation of abundance}

The resulting alignments were used to reconstruct transcript models using the software package Cufflinks (v2.1.1) [34]. This program constructs a parsimonious set of transcript models that best explain the read alignments observed in the samples. In addition, the tool cuffmerge was used for merging together each of the assemblies with the reference sheep annotation file in order to combine novel transcripts with known annotated transcripts. This strategy maximizes the overall quality of the final assembly. Finally, abundances of transcripts were scaled via the median of the geometric means of fragment counts across all libraries (as described in [35]) and corrected for sequence bias [36] in order to improve expression estimates.

\section{Overall gene expression analysis}

Differentially expressed genes were detected using Cuffdiff, a companion tool of Cufflinks. Cuffdiff performs 
differential analysis at both gene and transcript-level resolution and controls for both variability across replicates and uncertainty in abundance expression estimates caused by ambiguously mapped reads [37]. In addition, Cuffdiff controls for cross-replicate variability and also read-mapping ambiguity by using a model for fragment counts based on the beta negative binomial distribution [37]. Here, the analyses were performed using the default settings for all parameters. Finally, to account for multiple hypothesis testing and control the false discovery rate, $P$-values reported by Cuffdiff were corrected using the Benjamini-Hochberg procedure [38].

\section{Validation of differentially expressed genes}

Four genes that showed significant differential expression between $\mathrm{CN}$ and DG in subcutaneous adipose depot tissue were chosen for validation of the RNA-Seq results: ankyrin repeat domain 1 ( $A N K R D 1$, also known as $C A R P)$, kringle containing transmembrane protein 1 (KREMEN1), muscle-related coiled-coil protein (MURC), and synaptopodin 2-like (SYNPO2L). The RNA samples used for RNA-Seq were used here for validation of differential expression. A total of $1 \mu \mathrm{g}$ RNA from each sample was used to synthesize cDNA using the iScript cDNA Synthesis Kit (Bio-Rad Laboratories, Hercules, CA, USA) following the manufacturer's instructions. Quantitative real-time PCR (qRT-PCR) was used to estimate the fold change in expression between treatments. The ribosomal protein L19 (RPL19) gene was chosen as internal control because of its stable expression across samples. All samples were run in triplicates in the ECO real-time PCR system (Illumina, San Diego, CA) using iQSYBR Green Supermix (Bio-Rad Laboratories, CA). Primers were designed to cross exon-exon junctions to minimize the potential of amplifying genomic DNA and are shown in Table 2. The relative gene expression values were calculated using the $2^{-\Delta \Delta \mathrm{Ct}}$ method [39].

Table 2 Primers used for the validation of gene expression

\begin{tabular}{clc}
\hline Gene & Primer sequence $\mathbf{5}^{\prime} \rightarrow \mathbf{3}^{\prime}$ & Amplicon size $\mathbf{( b p )}$ \\
\hline ANKRD1 & F: GTATCTCCTTCCGGTCTTGG & 117 \\
& R: CGCGATAATTGCTCAGCAC & \\
KREMEN1 & F: GATGGCAGGGTCATCATCTTC & 67 \\
& R: GAGGTCATGGCCGAGTAATTC & \\
MURC & F: CCTCTGGACTCTCAATCAAGC & 93 \\
& R: AACAAATTCCGTGGGTCAT & \\
PRL19 & F: CAACTCCCGCCAGCAGAT & 76 \\
& R: CCGGGAATGGACAGTCACA & \\
SYNPO2L & F: CTGTGCGACCGCCACCCT \\
& R: GTGTTCAGGGATCCAGC & 115 \\
\hline
\end{tabular}

\section{Gene set enrichment analysis}

The significant enrichment of Gene Ontology (GO) terms and KEGG pathways with genes differentially expressed between maternal diets was analyzed using Fisher's exact test, a test of proportions based on the cumulative hypergeometric distribution [40]. For each comparison of interest, genes that showed a FDR $\leq 0.20$ and had ENSEMBL annotations were tested against the background set of all genes with ENSEMBL annotations. The procedure proposed by Benjamini and Hochberg [38] was applied in order to account for multiple testing. Functional categories with FDR $\leq 0.05$ were considered significant. These analyses were performed using the procedure FatiGO [41], implemented on the platform Babelomics [42], and using the Bioconductor packages org.Bt.eg.db, GO.db, and KEGG.db that are available in the $\mathrm{R}$ language/environment.

\section{Results}

Sequencing of the transcriptome of different fetal sheep tissues

To assess the effects of three different maternal diets on the transcriptome of three different fetal tissues in sheep, a total of 36 pooled samples (12 pooled samples per tissue with 4 biological replicates per diet) were analyzed using RNA-sequencing. Additional file 2 shows the results of the alignment of the sequencing reads to the sheep reference genome. Approximately, 30 million reads were sequenced for each sample. Sequencing reads were aligned against the recent ovine reference genome (Oar_v3.1) using the software package Tophat. On average, $83 \%$ of the total reads were successfully mapped by allowing no more than two mismatches and restricting the alignments to at most 40 genomic locations (Additional file 2). Among the aligned reads, approximately $86 \%$ and $65 \%$ were mapped to unique genomic regions in muscle and adipose tissues, respectively. Sequencing data can be accessed by GEO with the accession number GSE62938.

\section{Differential gene expression in longissimus dorsi muscle tissue}

A total of 18,393 genes were tested for differential expression between maternal diets in fetal longissimus dorsi muscle tissue. Marked differences in gene expression were found between $\mathrm{CN}$ diet and the other two maternal diets. Controlling FDR at 0.05 , a total of 224, 823 and 29 genes showed differential expression between $\mathrm{CN}$ vs. DG, CN vs. HY, and DG vs. HY, respectively (Figure 1A). Notably, the expression of most of these genes was decreased by maternal $\mathrm{CN}$ diet. In particular, 168 out of 224 genes, and 600 out of 823 genes showed higher expression in fetuses from DG and HY compared with those derived from $\mathrm{CN}$ diet, respectively (Figure 1A). Maternal diets DG and HY induced a very similar pattern 


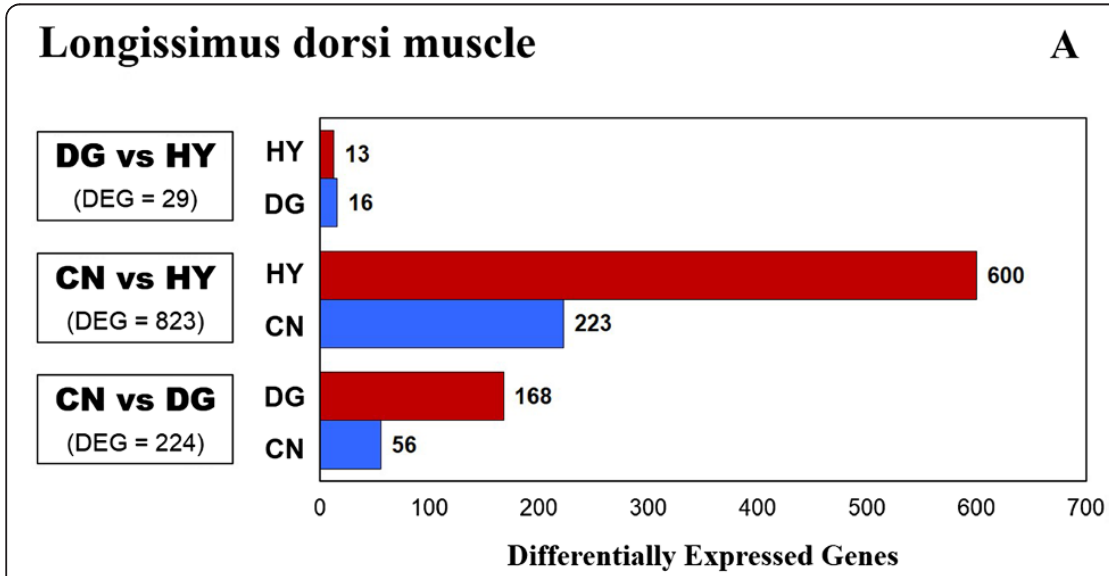

\section{Subcutaneous fat}

C
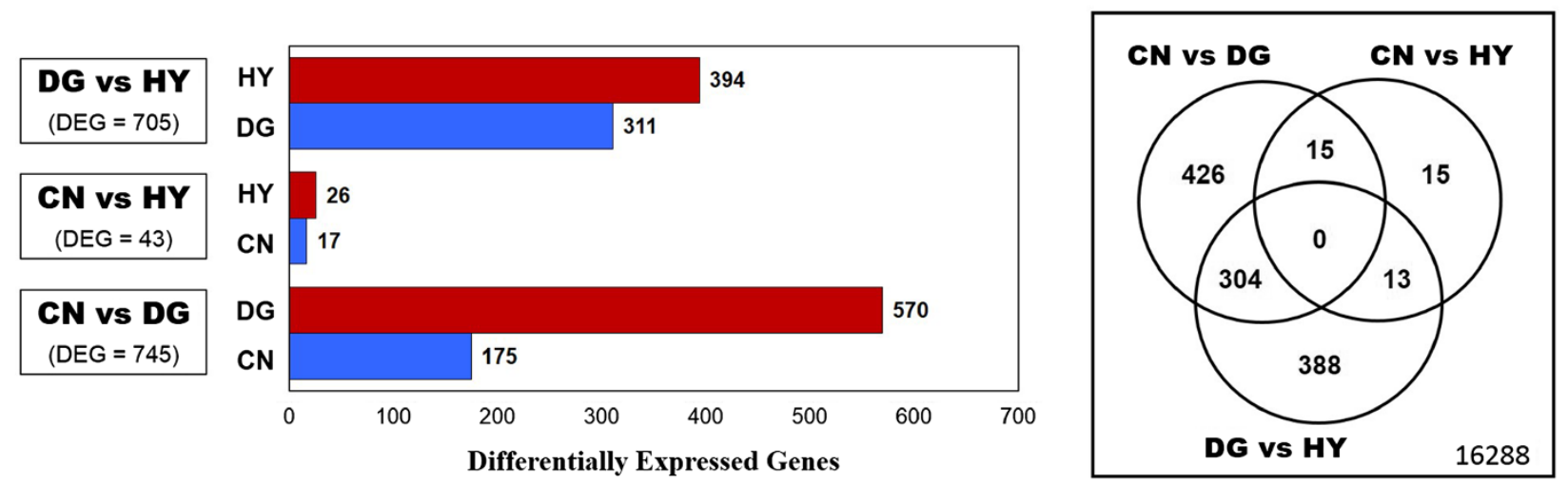

Perirenal fat

$\mathbf{E}$

F
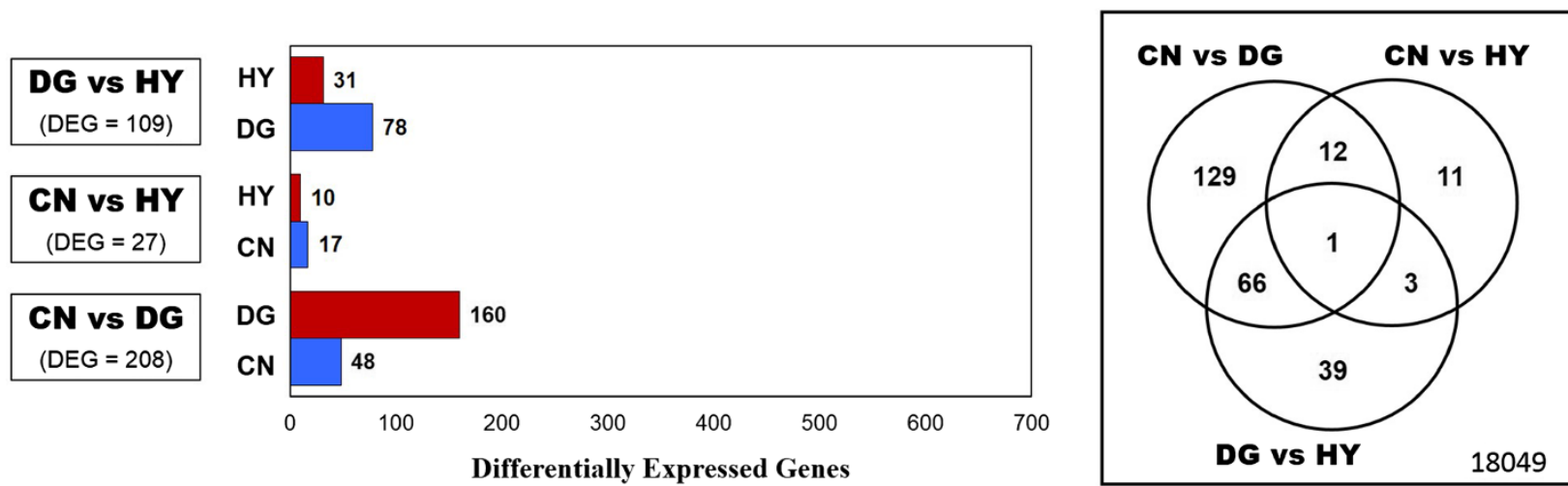

Figure 1 Comparison of overall gene expression between maternal diets in three different fetal tissues. Fetal tissues: longissimus dorsi (A-B), subcutaneous adipose depot (C-D), and perirenal adipose depot (E-F). Diets fed to dams: $\mathbf{H Y}=$ ad libitum fed alfalfa haylage; $\mathbf{C N}=$ limit-fed whole shell corn; DG = limit-fed corn dried distillers grains. The bar graphs show the number of genes up-regulated in each diet for each of the three pairwise comparisons. The Venn diagrams show the overlap between genes that showed significant differential expression in each of the three pairwise comparisons.

of gene expression in fetal muscle; indeed, only 29 genes showed differential expression at a FDR $\leq 0.05$ between these two diets and none of them were significant at a FDR $\leq 0.01$.
Figure 1B shows the overlap between genes that were differentially expressed in each of the three pairwise comparisons. Interestingly, a total of 166 genes differed simultaneously between $\mathrm{CN}$ diet and the other two 
maternal diets. Many of these genes are directly involved in embryonic and fetal development [e.g., ankyrin repeat domain 11 (ANKRD11), axin 1 (AXIN1), epsin 1 (EPN1), epsin 2 (EPN2), guanine nucleotide binding protein (G protein) alpha 12 (GNA12), methyl-CpG binding domain protein $3(M B D 3), \mathrm{WD}$ and tetratricopeptide repeats 1 (WDTC1)], skeletal muscle cell and tissue differentiation [e.g., ankyrin repeat domain 1 (ANKRD1), B cell CLL/ lymphoma 9-like $(B C L 9 L)$, histone cell cycle regulator (HIRA), myogenic differentiation 1 (MYOD1), sarcoglycan alpha $(S G C A)$, tripartite motif containing 72, E3 ubiquitin protein ligase (TRIM72)], and muscle myosin complex and sarcomere organization [e.g., myosin, heavy chain 13, skeletal muscle (MYH13), nebulin-related anchoring protein $(N R A P)$, parvin beta $(P A R V B)$, synaptopodin 2-like (SYNPO2L), titin-cap (TCAP)]. Importantly, the expression of all these genes was decreased in fetuses derived from dams fed $\mathrm{CN}$ diet (FDR $\leq 0.05$ ).

To gain insight into the biological processes that could be regulated differentially between maternal diets, we performed a gene set enrichment analysis. Many functional categories that were significantly enriched (FDR $\leq 0.05$ ) with differentially expressed genes were detected simultaneously in both $\mathrm{CN}$ vs. DG and $\mathrm{CN}$ vs. HY pairwise comparisons. In particular, GO terms structural constituent of ribosome, ribosome, translation, chromatin, and nucleosome assembly, and KEGG pathway ribosome were all significantly enriched (FDR $\leq 0.05)$ with genes differentially expressed in fetal muscle between $\mathrm{CN}$ and the other two maternal diets. We further characterized two relevant GO terms, chromatin and ribosome, in order to assess the general pattern of gene expression within these pathways. In the case of the GO term chromatin, the expression of eight of the nine significant genes was decreased in fetuses from $\mathrm{CN}$-fed dams. On the other hand, 26 out of 36 differentially expressed genes in the $\mathrm{GO}$ term ribosome were up-regulated in the muscle of fetuses derived from $\mathrm{CN}$ dietary treatment. Moreover, seven different KEGG pathways closely related to energetic metabolism, including glycolysis and gluconeogenesis, citrate cycle, valine, leucine and isoleucine degradation, propanoate metabolism, and insulin signaling pathway, showed significant enrichment (FDR $\leq 0.05$ ) of differentially expressed genes between $\mathrm{CN}$ and $\mathrm{HY}$ diets (Table 3). Notably, the expression of most genes within these pathways was decreased by maternal $\mathrm{CN}$ diet (Table 3).

Differential gene expression in subcutaneous adipose tissue Noticeable differences in gene expression in fetal subcutaneous adipose tissue were found between DG maternal dietary feed source and the other two maternal diets. Of the 17,449 genes evaluated for differential expression, a total of 745,705 and 43 genes showed differential
Table 3 KEGG pathways significantly enriched with differentially expressed genes (DEG) between $\mathrm{CN}$ and HY maternal diets in fetal longissimus dorsi muscle tissue

\begin{tabular}{|c|c|c|c|}
\hline KEGG term (ID) & $\begin{array}{c}\text { No. } \\
\text { genes }\end{array}$ & $\begin{array}{c}\text { No. DEG } \\
(\uparrow \mathrm{HY})\end{array}$ & $q$-value \\
\hline Glycolysis/Gluconeogenesis (00010) & 32 & $10(9)$ & 0.050 \\
\hline Citrate cycle (TCA cycle) (00020) & 23 & $10(10)$ & 0.018 \\
\hline Galactose metabolism (00052) & 18 & $7(7)$ & 0.050 \\
\hline Valine, (iso)leucine degradation (00280) & 32 & $14(13)$ & 0.001 \\
\hline Starch and sucrose metabolism (00500) & 19 & $8(8)$ & 0.035 \\
\hline Propanoate metabolism (00640) & 22 & $9(8)$ & 0.022 \\
\hline Ribosome (03010) & 48 & $22(3)$ & $<0.001$ \\
\hline Insulin signaling pathway (04910) & 104 & $25(23)$ & 0.035 \\
\hline
\end{tabular}

Diets fed to dams: $\mathbf{C N}=$ limit-fed whole shell corn; $\mathrm{HY}=$ ad libitum fed alfalfa haylage.

expression (FDR $\leq 0.05)$ between DG vs. CN, DG vs. HY, and $\mathrm{CN}$ vs. HY, respectively (Figure $1 \mathrm{C}$ ). Most of the significant genes showed higher expression in fetuses derived from DG-fed dams compared with $\mathrm{CN}$-fed dams, while similar proportion of highly and lowly expressed genes was found in the comparison between DG and HY diets (Figure $1 \mathrm{C}$ ). Interestingly, only 43 genes showed differential expression at a FDR $\leq 0.05$ between $\mathrm{CN}$ and $\mathrm{HY}$ dietary treatments indicating very similar pattern of overall gene expression (Figure 1C). None of these 43 genes was significant at a FDR $\leq 0.01$.

Among the differentially-expressed genes (FDR $\leq 0.05$ ) in DG vs. CN and DG vs. HY, a total of 304 genes were detected in both pairwise comparisons (Figure 1D). Many of these genes are closely related to embryonic and fetal development [e.g., angiomotin (AMOT), integrin, beta 6 (ITGB6), shisa family member 2 (SHISA2), SRY (sex determining region Y)-box 6 (SOX6), transforming growth factor, beta 2 (TGFB2), ubiquitin protein ligase E3 component n-recognin 3 (UBR3), WD and tetratricopeptide repeats 1 (WDTC1)], adipose tissue development [e.g., acetoacetyl-CoA synthetase $(A A C S)$, AT rich interactive domain 5B (MRF1-like) (ARID5B), patatin-like phospholipase domain containing 3 (PNPLA3)], cholesterol and fatty acid biosynthetic process [e.g., MLX interacting protein-like $(M L X I P L)$, protein kinase, AMP-activated, alpha 2 catalytic subunit (PRKAA2), protein kinase, AMPactivated, beta 2 non-catalytic subunit (PRKAB2)], and metabolism of lipids and lipoproteins [e.g., angiopoietinlike 4 (ANGPTL4), cell death-inducing DFFA-like effector A $(C I D E A)$, cell death-inducing DFFA-like effector $C$ $(C I D E C)$, carnitine palmitoyltransferase 1A (CPT1A), nuclear receptor subfamily 1 , group $\mathrm{D}$, member 2 (NR1D2), phospholipase A2, group VII (platelet-activating factor acetylhydrolase, plasma) (PLA2G7)]. The majority of these genes showed higher expression in the fetuses derived from dams fed DG diet. 
Gene set enrichment analysis was performed to further characterize the processes that could be regulated differentially in fetal subcutaneous fat due to maternal nutrition. Interestingly, we found three functional categories, GO terms dephosphorylation, phosphatase activity, and phosphoric ester hydrolase activity that were enriched (FDR $\leq 0.05)$ with significant genes detected in both pairwise comparisons involving DG maternal diet, i.e., DG vs. CN and DG vs. HY comparisons (Table 4). The majority of the genes in these pathways showed higher expression in fetuses from dams fed DG diet; this pattern is more noticeable in the GO term dephosphorylation (Table 4). Moreover, 10 different GO terms that belong to the biological process domain showed significant enrichment (FDR $\leq 0.05$ ) of differentially expressed genes detected between DG and CN maternal diets (Figure 2A). These functional categories are mainly involved in embryo and fetal development (e.g., tissue development, organ development, multicellular organismal development) and ion transport (e.g., cation transport, calcium ion transport, metal ion transport). Remarkably, the expression of most of the genes in these pathways was increased by maternal DG dietary treatment (Figure 2A).

\section{Differential gene expression in perirenal adipose tissue}

A total of 18,310 genes were evaluated for differential expression between maternal diets in fetal perirenal adipose tissue. Among the genes analyzed, 208, 109 and 27 genes showed differential expression (FDR $\leq 0.05$ ) between DG vs. CN, DG vs. HY, and CN vs. HY, respectively (Figure 1E). Marked differences were found only between DG and CN maternal diets; none of the other two pairwise comparisons detected significant genes at FDR $\leq 0.01$. Most of the significant genes showed higher expression in fetuses derived from dams fed DG diet.

We further characterized the 208 genes that showed differential expression (FDR $\leq 0.05$ ) between DG and CN maternal dietary feed sources. Many of these genes are associated with embryo and fetal development [e.g., integrin, beta 4 (ITGB4), latent transforming growth factor beta binding protein 4 (LTBP4), methyl-CpG binding domain protein $3(M B D 3)$, retinol binding protein 4 (RBP4), secreted frizzled-related protein 5 (SFRP5)], adipogenesis [e.g., CCAAT/enhancer binding protein $(\mathrm{C} / \mathrm{EBP})$, beta $(C E B P B)$, delta-like 1 homolog (Drosophila) (DLK1), lamin $\mathrm{A} / \mathrm{C}(L M N A)]$, and lipid metabolic process [e.g., acetyl-CoA acyltransferase $1(A C A A 1)$, apolipoprotein D $(A P O D)$, apolipoprotein $\mathrm{E}(A P O E)$, low density lipoprotein receptor-related protein 1 ( $L R P 1)$, prostaglandin I2 (prostacyclin) synthase (PTGIS)]. All these genes were upregulated in the perirenal adipose tissue of fetuses derived from dams fed the DG diet (FDR $\leq 0.05$ ).

Gene set enrichment analysis revealed $10 \mathrm{GO}$ terms from the biological process domain significantly enriched with genes differentially expressed between DG and CN maternal diets (Figure 2B). These GO terms are closely related to fetal development (e.g., tissue development, organ development, anatomical structure development), cellular adhesion (e.g., cell adhesion, cell-substrate adhesion) and Wnt signaling pathway. The expression of most genes in these functional categories was increased by maternal DG diet (Figure 2B). Interestingly, many of these significant GO terms, especially those related to tissue and organ development, were also enriched with significant genes detected between DG and $\mathrm{CN}$ maternal diets in fetal subcutaneous fat tissue.

\section{Validation of overall gene expression}

To validate genes found to be significant in the RNASeq analysis, four differentially expressed genes detected in fetal subcutaneous adipose tissue (ANKRD1, KRE$M E N 1, M U R C$, and SYNPO2L) were selected and their expression was assessed using qRT-PCR. RNA-Seq analysis revealed higher expression of these four genes in subcutaneous fat in fetuses derived from DG-fed dams compared with fetuses from $\mathrm{CN}$-fed dams. Figure 3 displays the fold differences in gene expression measured by both RNA-Seq and qRT-PCR. The four genes showed similar patterns of mRNA abundance with both methods (Figure 3).

\section{Discussion}

There is growing evidence that nutritional perturbations during different stages of pregnancy can modify the fetal development of skeletal muscle and adipose tissues, which in turn could have important implications in food animal production $[9,21,27,43,44]$. The present study was specifically designed to determine whether maternal

Table 4 Gene Ontology terms significantly enriched with differentially expressed genes (DEG) between DG and CN or HY maternal diets in fetal subcutaneous adipose tissue

\begin{tabular}{|c|c|c|c|c|c|c|}
\hline \multirow[t]{2}{*}{ GO term } & \multicolumn{3}{|c|}{ DG vs $\mathrm{CN}$} & \multicolumn{3}{|c|}{ DG vs HY } \\
\hline & No. genes & No. DEG ( $\uparrow \mathrm{DG})$ & q-value & No. genes & No. DEG ( $\uparrow \mathrm{DG})$ & q-value \\
\hline Dephosphorylation (0016311) & 106 & $24(21)$ & 0.025 & 108 & $29(21)$ & 0.035 \\
\hline Phosphatase activity (0016791) & 156 & $29(18)$ & 0.050 & 160 & $36(21)$ & 0.030 \\
\hline Phosphoric Ester Hydrolase Activity (0042578) & 199 & $36(25)$ & 0.043 & 205 & $46(27)$ & 0.008 \\
\hline
\end{tabular}

Diets fed to dams: HY = ad libitum fed alfalfa haylage; $C N=$ limit-fed whole shell corn; DG = limit-fed corn dried distiller's grains 


\section{A. Subcutaneous fat}

$$
\begin{array}{c|c|c|}
\hline \text { Dephosphorylation (0.025) } & \mathbf{2 1} & \mathbf{3} \\
\cline { 2 - 3 } & & \\
\text { Ion homeostasis (0.040) } & \mathbf{2 0} & \mathbf{7} \\
\cline { 2 - 3 }
\end{array}
$$

Chemical homeostasis (0.038) $21 \quad 12$

Metal ion transport (0.004) $\quad 400$

Calcium ion transport (0.003) 19

Cation transport (0.035) \begin{tabular}{|l|l|}
\hline 43 & 5 \\
\cline { 2 - 2 }
\end{tabular}

\begin{tabular}{l|l|l|}
\cline { 2 - 2 } Ion transport (0.002) & 55 & 10 \\
\cline { 2 - 2 }
\end{tabular}

Organ development (0.038)

78

Tissue development (0.005)

\begin{tabular}{|l|l|}
\hline 45 & 9 \\
\hline
\end{tabular}

Multicellular organismal development $(0.043)$

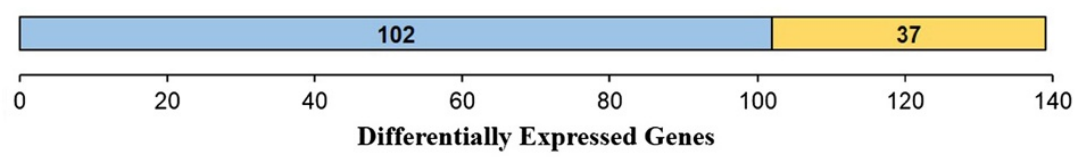

\section{B. Perirenal fat}

Wnt receptor signaling pathway $(0.005)$

Regulation of body fluid levels (0.003)

Extracellular matrix organization (0.001)

15

Cell-substrate adhesion (0.041)

\section{7}

Cell adhesion (0.001)

25 1

Anatomical structure development (0.002)

41

Organ morphogenesis $(0.034)$

16

2

Organ development (0.016)

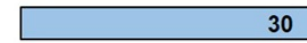

0 $\mid 3$

Tissue development (0.006) 17 2

Multicellular organismal development $(0.001)$

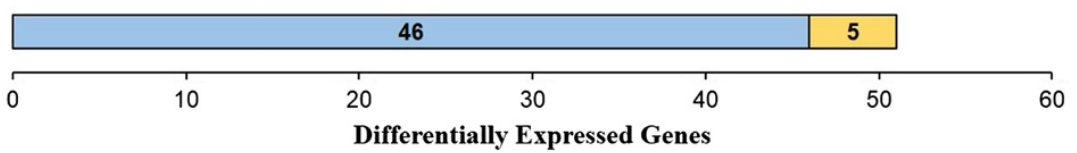

Figure 2 Gene Ontology biological process terms significantly enriched with differentially expressed genes between DG and CN maternal diets in fetal subcutaneous adipose tissue (A) and fetal perirenal adipose tissue (B). The graphs show the number of differentially expressed genes per each significant functional category (q-values). Genes up-regulated in DG diet are in blue while genes up-regulated in CN diet are in yellow. 


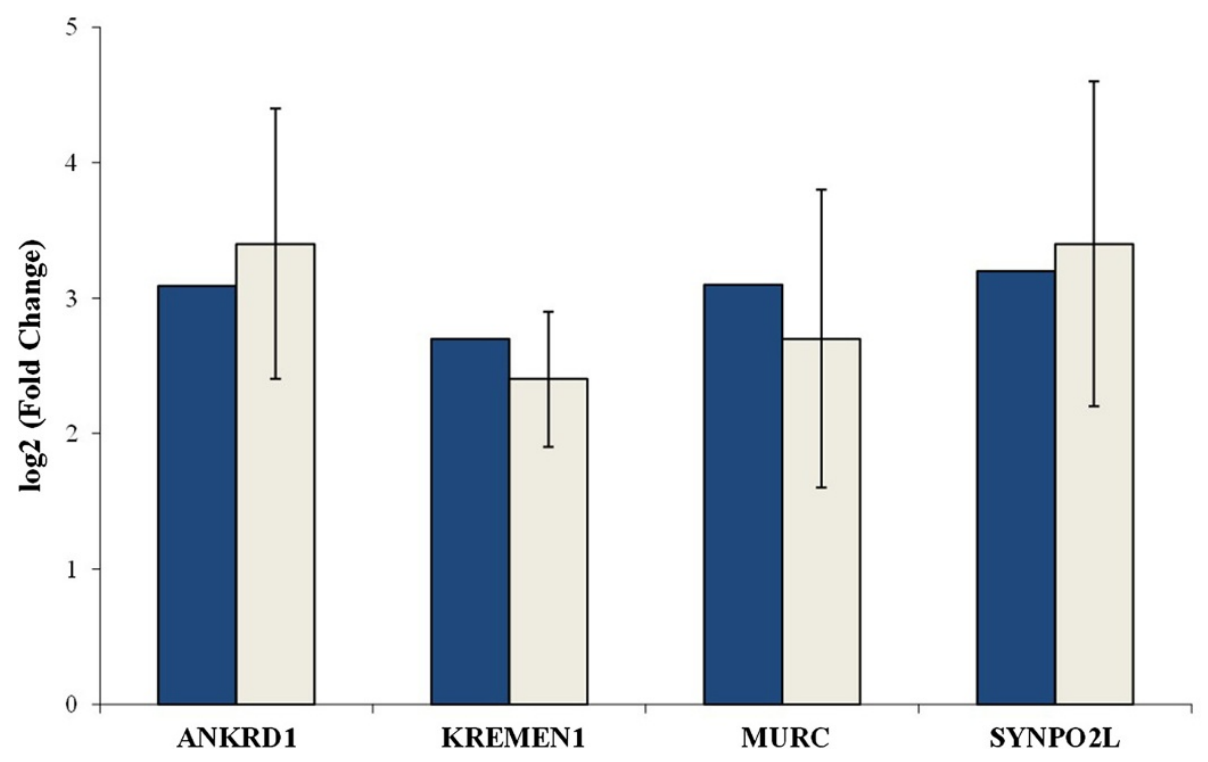

Figure 3 Fold changes of four differentially expressed genes measured by RNA-Seq (blue) versus qRT-PCR (light grey). The four genes show higher expression in subcutaneous fat in fetuses from DG diet compared with CN maternal diet.

diets differing in feed source and nutritive components during mid-to-late gestation impacts the transcriptome of fetal muscle and adipose tissues in sheep. Maternal diets, formulated to provide isoenergetic intakes, differed in the primary feed energy source. More specifically, for ewes fed HY, the energy source was primarily fiber while energy source for ewes fed $\mathrm{CN}$ was primarily starch, and for ewes fed DG, energy came from the combination of fiber, fat, and protein thus impacting the substrate supply to the fetus. Additionally, maternal diets differed in protein and fat content. Dams consuming the $\mathrm{CN}$ diet had a lower protein intake than dams consuming DG and HY diets, whereas dam consuming the DG diet had higher fat intake than dams consuming $\mathrm{CN}$ and $\mathrm{HY}$ feedstuffs (see Table 1). Remarkably, these prepartum diets caused notable changes in gene expression in fetal longissimus dorsi muscle, subcutaneous, and perirenal adipose tissues. In particular, maternal $\mathrm{CN}$ diet mainly impacted fetal muscle development while the DG diet mainly affected the development of fetal adipose depots. Hence, our findings support the idea that maternal nutrition during mid-to-late gestation can impact the programing of fetal muscle and fat tissues in sheep.

\section{Maternal nutrition impacts fetal tissue development including myogenesis and adipogenesis}

Remarkable gene expression differences in longissimus dorsi muscle were found between fetuses from $\mathrm{CN}$-fed dams and fetuses from either DG-fed or HY-fed dams (Figure 1A-B). Many of the most significantly altered genes are associated with skeletal muscle development. For instance, MYOD1 encodes a nuclear protein that belongs to the myogenic basic helix-loop-helix transcription factor family, and plays key roles in skeletal muscle cell specification and differentiation [45]. ANKRD1, a member of the muscle ankyrin repeat protein family, is a transcription factor involved in the signaling cascade during myogenic differentiation, myofibrillar assembly, and muscle remodeling [46]. HIRA is a replicationindependent histone chaperone that is linked to transcription and various developmental processes, including the activation of relevant genes during skeletal myogenesis [47]. BCL9 is an essential component of canonical Wnt signaling cascade that mediates myogenic differentiation during muscle development and regeneration [48]. Importantly, the expression of all these genes was decreased in fetuses derived from dams fed the $\mathrm{CN}$ diet. In addition, relevant genes associated with muscle myosin complex and sarcomere organization also showed differential gene expression due to $\mathrm{CN}$ maternal diet. For instance, NRAP is a muscle-specific scaffolding protein that is involved in myofibril assembly and sarcomere organization [49]. PARVB is localized at regions of the sarcolemma in skeletal muscle and plays both physical and signaling roles in muscle cells [50]. TCAP is a key component of the Z-disk in striated and cardiac muscle and has a relevant role in sarcomere assembly and integrity [51]. SYNPO2L is another component of the Z-disk with important roles in heart and skeletal muscle function and development [52]. These genes were also downregulated in the muscle of fetuses derived from $\mathrm{CN}$-fed dams. Overall, these results suggest that a prepartum maternal $\mathrm{CN}$ diet, i.e., starch-based diet, altered myogenesis, myofibrillogenesis and sarcomere organization in fetal 
longissimus dorsi muscle. Crude protein intake of ewes fed $\mathrm{CN}$ was less than that for ewes fed DG and HY. Interestingly, previous studies have shown that low protein maternal diets, compared against high protein diets fed at isocaloric intakes, adversely affected myogenesis, muscle differentiation, and muscle fiber number in newborn and fattening piglets $[53,54]$. Although it seems that a highstarch maternal diet during pregnancy does not affect muscle weights in sheep progeny at birth [31], the possible effect of this diet on morphological and histological parameters of the muscle remains unknown and warrants future studies.

Fetal adipose tissues also showed notable gene expression changes due to different maternal dietary treatments (Figure 1C-F). Among the most significant genes, many are closely related to adipose tissue development. For instance, $A R I D 5 B$, a member of the AT-rich interaction domain family of DNA binding proteins, regulates the transcription of key genes involved in adipogenesis, including adipogenic transcription factors $\mathrm{C} / \mathrm{EBP} \alpha$, and PPARY [55]. CEBPB, a member of CCAAT/enhancer binding protein family, encodes a transcription factor whose expression is induced directly by adipogenic hormones, and which plays essential early catalytic roles in the adipocyte differentiation program [56]. LMNA encodes an intermediate filament protein localized in the nuclear lamina and has crucial roles in early post-natal differentiation and maturation of adipose tissue [57]. All these genes showed higher expression in fat tissues from fetuses derived from DG-fed dams. In addition, gene set enrichment analysis revealed that $\mathrm{GO}$ functional categories related to tissue development and organogenesis, such as tissue development, organ development, and multicellular organismal development, were significantly enriched with differentially expressed genes (Figure 2). These gene expression changes were more pronounced between DG and $\mathrm{CN}$ diets, and in general, genes in these GO terms showed higher expression in fetuses derived from DG-fed dams. It is important to note that dams consuming the DG prepartum diet had greater protein and fat intake than dams consuming the $\mathrm{CN}$ diet, and hence our findings suggest that prepartum diets with greater fat and protein content may alter fetal adipogenesis and tissue development. Previous studies have investigated the impact that different prepartum diets have on fetal adipose tissues. Larson et al. [17] have shown that maternal protein supplementation during late gestation increased overall percentage of body fat in steer progeny. Similarly, Underwood et al. [58] have shown that a low protein maternal diet negatively impact subcutaneous adipose tissue deposition in beef. In contrast, Rehfeldt et al. [53] reported that either high or low protein maternal diets, fed at isocaloric intakes during pregnancy, reduced both subcutaneous and perirenal fat content in newborn piglets. Furthermore, there is evidence that ingestion of a high-fat diet during pregnancy induced hypertrophy and hyperplasia of fetal adipose tissue [59]. Interestingly, Radunz et al. [15] have shown that a prepartum diet based on dried distillers grains, similar to our DG maternal diet, tended to increase overall internal fat, including perirenal fat, in sheep progeny.

Of particular interest, the term Wnt receptor signaling pathway showed an overrepresentation of significant genes in fetal perirenal fat tissue (Figure 2B). The seven genes that revealed differential expression were upregulated in fetuses from DG-fed dams. Interestingly, these seven genes, AXIN1, DKK3, LRP1, LRP4, SFRP1, $S F R P 2$, and SFRP4, are all negative regulators of the Wnt receptor signaling pathway $[60,61]$. It is well established that the Wnt receptor signaling pathway inhibits adipogenesis and hence the repression of this pathway is crucial for adipocyte differentiation and fat tissue development [61,62]. Therefore, these findings provide further evidence of the potential impact of a DG maternal diet on fetal adipose tissue development.

Maternal nutrition alters metabolic processes in fetal tissues Prepartum maternal diets impacted the energetic metabolism of fetal muscle. These changes were more marked between fetuses from $\mathrm{CN}$-fed dams vs. HY-fed dams, i.e., starch-based vs. fiber-based maternal diets. Several genes involved in energetic metabolism, including those implicated in glycolysis and gluconeogenesis, citrate cycle, and valine, leucine and isoleucine degradation, were downregulated by maternal $\mathrm{CN}$ diet (Table 3 ). Similarly, a recent study has shown that a low protein prepartum diet compared against a high protein control diet when fed at isoenergetic intake in mice, caused downregulation of several pathways related to energetic metabolism processes including glycolysis, citrate cycle, and amino acid degradation, in skeletal muscle in newborn offspring [63]. Additionally, fetuses derived from HY-fed dams showed increased expression of genes involved in propanoate metabolism and insulin signaling pathway (Table 3). Overall, these findings show that prepartum maternal isoenergetic diets, differing in the primary feed source, alter the energetic metabolism of fetal muscle.

Maternal dietary treatments also perturbed different biological and molecular processes in fetal adipose tissues. For instance, in subcutaneous adipose tissue, three functional categories closely related to metabolism of phosphate anhydrides and esters showed overrepresentation of differentially expressed genes (Table 4). GO terms dephosphorylation, phosphatase activity, and phosphoric ester hydrolase activity were mainly enriched with genes that showed significant higher expression in fetuses derived from DG-fed dams. Many relevant biochemical reactions in adipose tissue, including the formation of acyl 
CoA during the synthesis of fatty acids, involved phosphate anhydrides and esters. Other important processes, such as ion transport in subcutaneous fat depot and cell adhesion in perirenal fat tissue, were also altered by prepartum maternal diets, showing an up-regulation of gene expression induced by a DG diet (Figure 2). It is well documented that each fat depot has its own particular features, including differences in growth, cellularity and metabolism [64]. This may explain why maternal DG diet altered mainly metabolism processes in subcutaneous fat while it affected mainly cellularity and tissue integrity in perirenal fat depot.

\section{Potential mechanisms underlying the observed transcriptomic changes}

There is growing evidence that maternal nutrition can induce epigenetic alterations to change gene expression in the fetal genome, such as DNA methylation or histone modifications including methylation, acetylation, and phosphorylation [65]. These modifications depend on the availability of key compounds, such as methyl donors, supplied by different amino acids and vitamins present in the maternal diet. Indeed, this link between maternal nutrition and subsequent modification of gene expression in the fetal genome is one of the important molecular mechanisms proposed to explain the phenomenon of fetal programming [65-68]. We have recently reported that maternal DG and HY diets were associated with altered DNA methylation and expression of the imprinted genes IGF2R and H19 in fetal sheep tissues compared to maternal $\mathrm{CN}$ diet [32]. Interestingly, while DNA methylation at the gene promoter suppressed expression, gene body methylation was positively associated with gene expression [32]. Here, two functional terms related to chromatin biology and epigenetics, chromatin and nucleosome assembly, were altered in fetal muscle showing significantly lower expression in fetuses from $\mathrm{CN}$-fed dams. Many genes from these pathways, such as H1FX, HIST2H2BE, MBD3, and SATB1, were also differentially expressed in fetal adipose tissues, showing lower expression in fetuses from maternal $\mathrm{CN}$ diet. Of special interest is $M B D 3$, an essential member of the nucleosome remodeling and deacetylation (NuRD) complex, a multi-functional complex which has key roles in chromatin remodeling during development [69]. MBD3 is associated with sites in the genome with activated genes, i.e., sites devoid of methylated CpG islands and with chromatin modifications that are characteristic of an active chromatin state [70]. Although DNA methylation or histone modifications were not directly evaluated in our experiment, it seems likely that maternal diets altered chromatin marks of key regions of DNA and these changes probably underlie the transcriptomic changes observed in fetal tissues.

\section{Conclusions}

The present study has characterized the effects of different maternal feed sources during mid-to-late gestation on the transcriptome of fetal tissues in sheep. These prepartum maternal diets caused notable gene expression changes in fetal adipose and muscle tissues. In particular, a maternal starch-based diet mainly altered fetal muscle development while a maternal diet with high fiber, protein, and fat concentrations mainly impacted fetal subcutaneous and perirenal adipose tissues. These findings provide evidence that maternal nutrition during mid-tolate gestation can perturb the programming of fetal muscle and fat tissues in sheep, which in turn may have important implications in food animal production. Determination of the potential effects of these maternal diets on epigenetic modifications in the fetal genome from these tissues is needed in future studies. In sum, the functional ramifications of the observed changes in gene expression, in terms of postnatal growth, body composition, and meat quality of the offspring, also warrant future investigation.

\section{Additional files}

Additional file 1: Description of the pools in terms of number of fetuses and their dams.

Additional file 2: Summary of sequencing read alignments to the reference genome.

\section{Competing interests}

The authors declare that they have no competing interests.

\section{Authors' contributions}

$H K, F P$, and AER designed the study. AER, XW, and FP performed experiments. FP and GJMR analyzed the data. FP wrote the manuscript. All authors read and approved the final manuscript.

\section{Acknowledgements}

This research was funded by USDA Hatch act fund 142-PRJ43 from the University of Wisconsin to AER and HK. FP was supported by the Agriculture and Food Research Initiative Competitive Grant no. 2011-67015-30219 from the USDA National Institute of Food and Agriculture to GJMR.

\section{Author details}

${ }^{1}$ Department of Animal Sciences, University of Wisconsin-Madison, 1675 Observatory Drive, Madison, WI 53706, USA. ${ }^{2}$ College of Animal Science and Technology, Northwest A\&F University, Yangling, Shaanxi 712100, China. ${ }^{3}$ Department of Animal and Food Science, University of Wisconsin-River Falls, River Falls, WI 54022, USA.

Received: 19 September 2014 Accepted: 19 November 2014 Published: 28 November 2014

\section{References}

1. Godfrey KM, Barker DJ: Fetal programming and adult health. Public Health Nutr 2001, 4:611-624.

2. Wu GY, Bazer FW, Cudd TA, Meininger CJ, Spencer TE: Maternal nutrition and fetal development. J Nutr 2004, 134:2169-2172.

3. Symonds ME, Stephenson T, Gardner DS, Budge H: Long-term effects of nutritional programming of the embryo and fetus: mechanisms and critical windows. Reprod Fertil Dev 2007, 19:53-63. 
4. Roseboom TJ, van der Meulen JHP, Ravelli ACJ, Osmond C, Barker DJP, Bleker OP: Effects of prenatal exposure to the Dutch famine on adult disease in later life: an overview. Mol Cell Endocrinol 2001, 185:93-98.

5. Painter RC, Roseboom TJ, Bleker OP: Prenatal exposure to the Dutch famine and disease in later life: an overview. Reprod Toxicol 2005, 20:345-352.

6. Woodall SM, Johnston BM, Breier BH, Gluckman PD: Chronic maternal undernutrition in the rat leads to delayed postnatal growth and elevated blood pressure of offspring. Pediatr Res 1996, 40:438-443.

7. Woods $L L$, Ingelfinger $J R$, Nyengaard JR, Rasch R: Maternal protein restriction suppresses the newborn renin-angiotensin system and programs adult hypertension in rats. Pediatr Res 2001, 49:460-467.

8. Guo F, Jen KLC: High-fat feeding during pregnancy and lactation affects offspring metabolism in rats. Physiol Behav 1995, 57:681-686.

9. Funston RN, Larson DM, Vonnahme KA: Effects of maternal nutrition on conceptus growth and offspring performance: implications for beef cattle production. J Anim Sci 2010, 88:E205-E215.

10. Wu G, Bazer FW, Wallace JM, Spencer TE: Board-invited review: intrauterine growth retardation: implications for the animal sciences. J Anim Sci 2006, 84:2316-2337.

11. Radunz AE, Fluharty FL, Relling AE, Felix TL, Shoup LM, Zerby HN, Loerch SC: Prepartum dietary energy source fed to beef cows: II. Effects on progeny postnatal growth, glucose tolerance, and carcass composition. J Anim Sci 2012, 90:4962-4974.

12. Micke GC, Sullivan TM, McMillen IC, Gentili S, Perry VEA: Heifer nutrient intake during early- and mid-gestation programs adult offspring adiposity and mRNA expression of growth-related genes in adipose depots. Reproduction 2011, 141:697-706.

13. Micke GC, Sullivan TM, McMillen IC, Gentili S, Perry VEA: Protein intake during gestation affects postnatal bovine skeletal muscle growth and relative expression of IGF1, IGF1R, IGF2 and IGF2R. Mol Cell Endocrinol 2011, 332:234-241.

14. Micke GC, Sullivan TM, Gatford KL, Owens JA, Perry VEA: Nutrient intake in the bovine during early and mid-gestation causes sex-specific changes in progeny plasma IGF-I, liveweight, height and carcass traits. Anim Reprod Sci 2010, 121:208-217.

15. Radunz AE, Fluharty FL, Susin I, Felix TL, Zerby HN, Loerch SC: Winter-feeding systems for gestating sheep II: effects on feedlot performance, glucose tolerance, and carcass composition of lamb progeny. J Anim Sci 2011, 89:478-488

16. Martin JL, Vonnahme KA, Adams DC, Lardy GP, Funston RN: Effects of dam nutrition on growth and reproductive performance of heifer calves. J Anim Sci 2007, 85:841-847.

17. Larson DM, Martin JL, Adams DC, Funston RN: Winter grazing system and supplementation during late gestation influence performance of beef cows and steer progeny. J Anim Sci 2009, 87:1147-1155.

18. Encinias HB, Lardy GP, Encinias AM, Hauer ML: High linoleic acid safflower seed supplementation for gestating ewes: effects on ewe performance, lamb survival, and brown fat stores. J Anim Sci 2004, 82:3654-3661.

19. Loerch SC: Limit-feeding corn as an alternative to hay for gestating beef cows. J Anim Sci 1996, 74:1211-1216.

20. Radunz AE, Fluharty FL, Day ML, Zerby HN, Loerch SC: Prepartum dietary energy source fed to beef cows: I. Effects on pre- and postpartum cow performance. J Anim Sci 2010, 88:2717-2728.

21. Du M, Tong J, Zhao J, Underwood KR, Zhu M, Ford SP, Nathanielsz PW: Fetal programming of skeletal muscle development in ruminant animals. J Anim Sci 2010, 88:E51-E60.

22. Du M, Huang Y, Das AK, Yang Q, Duarte MS, Dodson MV, Zhu MJ: Meat Science and Muscle Biology Symposium: manipulating mesenchymal progenitor cell differentiation to optimize performance and carcass value of beef cattle. J Anim Sci 2013, 91:1419-1427.

23. Greenwood PL, Slepetis RM, Bell AW, Hermanson JW: Intrauterine growth retardation is associated with reduced cell cycle activity, but not myofibre number, in ovine fetal muscle. Reprod Fertil Dev 1999, 11:281-291.

24. Fahey AJ, Brameld JM, Parr T, Buttery PJ: The effect of maternal undernutrition before muscle differentiation on the muscle fiber development of the newborn lamb. J Anim Sci 2005, 83:2564-2571.

25. Costello PM, Rowlerson A, Astaman NA, Anthony FE, Sayer AA, Cooper C, Hanson MA, Green LR: Peri-implantation and late gestation maternal undernutrition differentially affect fetal sheep skeletal muscle development. J Physiol-London 2008, 586:2371-2379.
26. Symonds ME, Pearce S, Bispham J, Gardner DS, Stephenson T: Timing of nutrient restriction and programming of fetal adipose tissue development. Proc Nutr Soc 2004, 63:397-403

27. Mostyn A, Symonds ME: Early programming of adipose tissue function: a large-animal perspective. Proc Nutr Soc 2009, 68:393-400.

28. Desai M, Ross MG: Fetal programming of Adipose tissue: effects of intrauterine growth restriction and maternal obesity/high-fat diet. Semin Reprod Med 2011, 29:237-245.

29. Gnanalingham MG, Mostyn A, Symonds ME, Stephenson T: Ontogeny and nutritional programming of adiposity in sheep: potential role of glucocorticoid action and uncoupling protein-2. Am J Physiol Regul Integr Comp Physiol 2005, 289:R1407-R1415.

30. Muhlhausler BS, Duffield JA, McMillen IC: Increased maternal nutrition stimulates peroxisome proliferator activated receptor-gamma, adiponectin, and leptin messenger ribonucleic acid expression in adipose tissue before. Endocrinology 2007, 148:878-885.

31. Radunz AE, Fluharty FL, Zerby HN, Loerch SC: Winter-feeding systems for gestating sheep I. Effects on pre- and postpartum ewe performance and lamb progeny preweaning performance. J Anim Sci 2011, 89:467-477.

32. Lan X, Cretney EC, Kropp J, Khateeb K, Berg M, Peñagaricano F, Magness R, Radunz A, Khatib H: Maternal diet during pregnancy induces gene expression and DNA methylation changes in fetal tissues in sheep. Front Genet 2013, 4:49.

33. Trapnell C, Pachter L, Salzberg SL: TopHat: discovering splice junctions with RNA-Seq. Bioinformatics 2009, 25:1105-1111.

34. Trapnell C, Williams BA, Pertea G, Mortazavi A, Kwan G, van Baren MJ, Salzberg SL, Wold BJ, Pachter L: Transcript assembly and quantification by RNA-Seq reveals unannotated transcripts and isoform switching during cell differentiation. Nat Biotechnol 2010, 28:511-518.

35. Anders $S$, Huber W: Differential expression analysis for sequence count data. Genome Biology 2010, 11:R106.

36. Roberts A, Trapnell C, Donaghey J, Rinn JL, Pachter L: Improving RNA-Seq expression estimates by correcting for fragment bias. Genome Biology 2011, 12:R22.

37. Trapnell C, Hendrickson DG, Sauvageau M, Goff L, Rinn JL, Pachter L: Differential analysis of gene regulation at transcript resolution with RNA-seq. Nat Biotechnol 2013, 31:562-578.

38. Benjamini $Y$, Hochberg $Y$ : Controlling the false discovery rate - a practical and powerful approach to multiple testing. J R Stat Soc Series B Stat Methodol 1995, 57:289-300.

39. Livak KJ, Schmittgen TD: Analysis of relative gene expression data using real-time quantitative PCR and the $2^{-D D C T}$ method. Methods 2001, 25:402-408

40. Peñagaricano F, Weigel KA, Rosa GJM, Khatib H: Inferring quantitative trait pathways associated with bull fertility from a genome-wide association study. Front Genet 2013, 3:307.

41. Al-Shahrour F, Diaz-Uriarte R, Dopazo J: FatiGO: a web tool for finding significant associations of Gene Ontology terms with groups of genes. Bioinformatics 2004, 20:578-580.

42. Medina I, Carbonell J, Pulido L, Madeira SC, Goetz S, Conesa A, Tarraga J, Pascual-Montano A, Nogales-Cadenas R, Santoyo J, García F, Marbà M, Montaner D, Dopazo J: Babelomics: an integrative platform for the analysis of transcriptomics, proteomics and genomic data with advanced functional profiling. Nucleic Acids Res 2010, 38:W210-W213.

43. McMillen IC, Muhlhausler BS, Duffield JA, Yuen BSJ: Prenatal programming of postnatal obesity: fetal nutrition and the regulation of leptin synthesis and secretion before birth. Proc Nutr Soc 2004, 63:405-412.

44. McMillen IC, Rattanatray L, Duffield JA, Morrison JL, MacLaughlin SM, Gentili S, Muhlhausler BS: The early origins of later obesity: pathways and mechanisms. In Advances in Experimental Medicine and Biology, Volume 646; 2009:71-81.

45. Rudnicki MA, Jaenisch R: The MyoD family of transcription factors and skeletal myogenesis. Bioessays 1995, 17:203-209.

46. Kojic S, Radojkovic D, Faulkner G: Muscle ankyrin repeat proteins: their role in striated muscle function in health and disease. Crit Rev Clin Lab SCl 2011, 48:269-294.

47. Yang JH, Choi JH, Jang H, Park JY, Han JW, Youn HD, Cho EJ: Histone chaperones cooperate to mediate Mef2-targeted transcriptional regulation during skeletal myogenesis. Biochem Biophys Res Commun 2011, 407:541-547.

48. Brack AS, Murphy-Seiler F, Hanifi J, Deka J, Eyckerman S, Keller C, Aguet M, Rando TA: BCL9 is an essential component of canonical Wnt signaling 
that mediates the differentiation of myogenic progenitors during muscle regeneration. Dev Biol 2009, 335:93-105.

49. Dhume A, Lu SJ, Horowits R: Targeted disruption of N-RAP gene function by RNA interference: a role for N-RAP in myofibril organization. Cell Motil Cytoskeleton 2006, 63:493-511.

50. Sepulveda JL, Wu C: The parvins. Cell Mol Life Sci 2006, 63:25-35.

51. Gregorio CC, Trombitas K, Centner T, Kolmerer B, Stier G, Kunke K, Suzuki K, Obermayr F, Herrmann B, Granzier H, Sorimachi H, Labeit S: The NH2 terminus of titin spans the Z-disc: its interaction with a novel 19-kD ligand (T-cap) is required for sarcomeric integrity. J Cell Biol 1998, 143:1013-1027.

52. Beqqali A, Monshouwer-Kloots J, Monteiro R, Welling M, Bakkers J, Ehler E, Verkleij A, Mummery C, Passier R: CHAP is a newly identified Z-disc protein essential for heart and skeletal muscle function. J Cell Sci 2010, 123:1141-1150.

53. Rehfeldt C, Lefaucheur L, Block J, Stabenow B, Pfuhl R, Otten W, Metges CC, Kalbe C: Limited and excess protein intake of pregnant gilts differently affects body composition and cellularity of skeletal muscle and subcutaneous adipose tissue of newborn and weanling piglets. Eur J Nutr 2012, 51:151-165.

54. Rehfeldt C, Stabenow B, Pfuhl R, Block J, Nurnberg G, Otten W, Metges CC Kalbe C: Effects of limited and excess protein intakes of pregnant gilts on carcass quality and cellular properties of skeletal muscle and subcutaneous adipose tissue in fattening pigs. J Anim Sci 2012, 90:184-196.

55. Yamakawa T, Whitson RH, Li SL, Itakura K: Modulator recognition factor-2 is required for adipogenesis in mouse embryo fibroblasts and 3 T3-L1 cells. Mol Endocrinol 2008, 22:441-453.

56. Lane MD, Tang QQ, Jiang MS: Role of the CCAAT enhancer binding proteins (C/EBPs) in adipocyte differentiation. Biochem Biophys Res Commun 1999, 266:677-683.

57. Kubben $\mathrm{N}$, Voncken JW, Konings $\mathrm{G}$, van Weeghel M, van den Hoogenhof MMG, Gijbels M, van Erk A, Schoonderwoerd K, van den Bosch B, Dahlmans V, Calis C, Houten SM, Misteli T, Pinto YM: Post-natal myogenic and adipogenic developmental Defects and metabolic impairment upon loss of A-type lamins. Nucleus-Austin 2011, 2:195-207.

58. Underwood KR, Tong JF, Price PL, Roberts AJ, Grings EE, Hess BW, Means WJ, Du M: Nutrition during mid to late gestation affects growth, adipose tissue deposition, and tenderness in cross-bred beef steers. Meat Sci 2010, 86:588-593.

59. Murabayashi N, Sugiyama T, Zhang L, Kamimoto Y, Umekawa T, Ma N, Sagawa N: Maternal high-fat diets cause insulin resistance through inflammatory changes in fetal adipose tissue. Eur J Obstet Gynecol Reprod Biol 2013, 169:39-44.

60. Kawano Y, Kypta R: Secreted antagonists of the Wnt signalling pathway. J Cell Sci 2003, 116:2627-2634.

61. Christodoulides C, Lagathu C, Sethi JK, Vidal-Puig A: Adipogenesis and WNT signalling. Trends Endocrinol Metab 2009, 20:16-24.

62. MacDougald OA, Mandrup S: Adipogenesis: forces that tip the scales. Trends Endocrinol Metab 2002, 13:5-11.

63. Mortensen $\mathrm{OH}$, Olsen HL, Frandsen L, Nielsen PE, Nielsen FC, Grunnet N, Quistorff B: A maternal low protein diet has pronounced effects on mitochondrial gene expression in offspring liver and skeletal muscle; protective effect of taurine. J Biomed Sci 2010, 17(Suppl 1):S38.

64. Hausman DB, DiGirolamo M, Bartness TJ, Hausman GJ, Martin RJ: The biology of white adipocyte proliferation. Obesity Rev 2001, 2:239-254.

65. Burdge GC, Lillycrop KA: Nutrition, epigenetics, and developmental plasticity: implications for understanding human disease. Annu Rev Nutr 2010, 30:315-339.

66. Burdge GC, Hanson MA, Slater-Jefferies JL, Lillycrop KA: Epigenetic regulation of transcription: a mechanism for inducing variations in phenotype (fetal programming) by differences in nutrition during early life? Br J Nutr 2007, 97:1036-1046.

67. Chmurzynska A: Fetal programming: link between early nutrition, DNA methylation, and complex diseases. Nutr Rev 2010, 68:87-98.

68. Tosh DN, Fu Q, Callaway CW, McKnight RA, McMillen IC, Ross MG, Lane RH, Desai M: Epigenetics of programmed obesity: alteration in IUGR rat hepatic IGF1 mRNA expression and histone structure in rapid vs. delayed postnatal catch-up growth: American Journal of Physiology-Gastrointestinal and Liver. Physiology 2010, 299:G1023-G1029.
69. Ho L, Crabtree GR: Chromatin remodelling during development. Nature 2010, 463:474-484

70. Gunther K, Rust M, Leers J, Boettger T, Scharfe M, Jarek M, Bartkuhn M, Renkawitz R: Differential roles for MBD2 and MBD3 at methylated CpG islands, active promoters and binding to exon sequences. Nucleic Acids Res 2013, 41:3010-3021.

doi:10.1186/1471-2164-15-1034

Cite this article as: Peñagaricano et al:: Maternal nutrition induces gene expression changes in fetal muscle and adipose tissues in sheep. BMC Genomics 2014 15:1034

\section{Submit your next manuscript to BioMed Central and take full advantage of:}

- Convenient online submission

- Thorough peer review

- No space constraints or color figure charges

- Immediate publication on acceptance

- Inclusion in PubMed, CAS, Scopus and Google Scholar

- Research which is freely available for redistribution 